\title{
Linear spaces with significant characteristic prime
}

go back

full screen

close

quit

\begin{abstract}
Let $G$ be a group with socle a simple group of Lie type defined over the finite field with $q$ elements where $q$ is a power of the prime $p$. Suppose that $G$ acts transitively upon the lines of a linear space $\mathcal{S}$. We show that if $p$ is significant then $G$ acts flag-transitively on $\mathcal{S}$ and all examples are known.
\end{abstract}

Keywords: linear space, group of Lie type, line-transitive, parabolic subgroup MSC 2000: 20B25, 05B05

\section{Background and statement of result}

A linear space $\mathcal{S}$ is an incidence structure of points and lines such that any two points are incident with exactly one line. Also $\mathcal{S}$ is non-trivial provided every line contains at least three points and there are at least two lines; all linear spaces considered in this paper will be presumed to be non-trivial. A flag is a pair $(\alpha, L)$ where $\alpha$ is a point incident with a line $L$.

Let $\mathcal{S}$ be a finite linear space admitting an automorphism group $G$ which is transitive on lines. Then $\mathcal{S}$ is said to have parameters $b$ (the number of lines), $v$ (the number of points), $k$ (the number of points incident with a line) and $r$ (the number of lines incident with a point).

Camina, Neumann and Praeger [?] have defined a prime $p$ to be significant for the space $\mathcal{S}$ if it divides into $(b, v-1)$. They then show that if $P$ is a Sylow $p$-subgroup of $G$ and $G_{\alpha}$ is a point-stabilizer in $G$ then $G_{\alpha} \geq N_{G}(P)$ [?, Lemma 6.1].

The finite linear spaces which admit a flag-transitive almost simple group have been classified in [?, ?]. As part of the program to extend this classification 




\section{The point stabilizer is non-maximal}

Lemma 3.1. Suppose that $G$ is a simple Chevalley group acting on a linear space $\mathcal{S}$ with $G_{\alpha}$ a non-maximal parabolic subgroup of $G$. Then a line stabilizer, $G_{\mathfrak{L}}$, is a parabolic subgroup of $G$ and $p$ is not significant.

Proof. Let $\Phi^{+}$be the set of positive roots associated with $G$ so that

$$
U=\prod_{s \in \Phi^{+}} X_{s}
$$

For $r \in \Pi$ be a fundamental root define the group $U_{r}=\prod_{s \in \Phi^{+} \backslash\{r\}} X_{s}$.

Now suppose that $G_{\alpha}$ is the parabolic subgroup $P_{J}$ where $J$ is a subset of the set of fundamental roots $\Pi$. Since $G_{\alpha}$ is non-maximal in $G$ we know that at least two fundamental roots, say $s$ and $t$, do not lie in $J$.

For $s$ a fundamental root recall the standard homomorphism $\phi_{s}$ from $\operatorname{SL}(2, q)$ into $\left\langle X_{s}, X_{-s}\right\rangle$. Then

$$
n_{s}:=\phi_{r}\left(\begin{array}{cc}
0 & 1 \\
-1 & 0
\end{array}\right)
$$

Now $n_{s}$ is an involution lying outside of $G_{\alpha}$ but which normalizes $U_{s}$ inside of $G_{\alpha}$. Hence $U_{s}$ fixes at least two points and hence the line between them. So $G_{\mathfrak{L}}$ contains a $G$-conjugate of $U_{s}$. Similarly $G_{\mathfrak{L}}$ contains a $G$-conjugate of $U_{t}$. In fact $G_{\mathfrak{L}}$ contains a $G$-conjugate of $U_{s}: H$ and $U_{t}: H$.

Now consider a Sylow $p$-subgroup of $G_{\mathfrak{L}}$. For some choice of $\mathfrak{L}$ this lies inside $U$. Now observe that, since $G=B N B$ and since both $U_{s}$ and $U$ are normal in $B$,

$$
\begin{aligned}
& U_{s}^{g}<U \\
\Longrightarrow & b_{1} n b_{2} U_{s} b_{2}^{-1} n^{-1} b_{1}^{-1}<U \text { where } g=b_{1} n b_{2} \\
\Longrightarrow & n U_{s} n^{-1}<U \\
\Longrightarrow & n U_{s} n^{-1}=U_{s} .
\end{aligned}
$$

Thus $U$ only contains one $G$-conjugate of $U_{s}$ and one $G$-conjugate of $U_{t}$, namely themselves. Furthermore they generate $U$. Thus $G_{\mathfrak{L}}$ contains $B=U: H$ as required.

Now $p$ does not divide into $b$ and so $p$ is not significant.

Lemma 3.2. Suppose that $G$ is a twisted simple group acting on a linear space $\mathcal{S}$ with $G_{\alpha}$ a non-maximal parabolic subgroup of $G$. Then $G_{\mathfrak{L}}$ is a parabolic subgroup of $G$ and $p$ is not significant. 

For the twisted groups this argument does not work in all cases. We need to show that $U_{J}^{1}: H$ is maximal in all conjugates of the Borel of which it is a subgroup. It is sufficient to show that $H$ acts transitively upon the set of nonidentity elements of $X_{J}^{1}$. We refer to [?, Tables 2.4 and 2.4.7] to see that this is only true when $X_{J}^{1}$ is of type I, II, III and VI as listed there. The cases we have excluded are when $G={ }^{2} \mathrm{~A}_{n}(q), n$ even, with $G_{\alpha}={ }^{\wedge}\left[q^{\frac{n^{2}+4 n}{4}}\right]: \mathrm{GL}_{\frac{n}{2}}\left(q^{2}\right)$; and when $G={ }^{2} \mathrm{~F}_{4}(q)$ with $G_{\alpha}=\left[q^{22}\right]: \mathrm{GL}_{2}\left(q^{2}\right)$.

Now we will investigate the possibility that there exists $g \in G_{\mathfrak{L}} \backslash\left(P_{\Pi \backslash K^{\prime}} \cap G_{\mathfrak{L}}\right)$. Suppose that this is the case. Since we have a $B N$-pair we can write $g=u_{1} n_{w} u$ where $u_{1}, u \in U$ and $n_{w} \in N$ maps onto $w \in W$ under the natural epimorphism. In fact, since $G_{\mathfrak{L}} \geq U_{r} H$ we can assume that $g=x_{r}(t) n_{w} x_{r}(u)$ where $t, u$ are elements of the finite field of order $q$.

Now suppose that $w(r) \neq \pm r$ (and note that then $w^{-1}(r) \neq \pm r$ ). We seek to prove the following

$$
g^{-1} U_{r} g \cap\left\langle U_{r}, X_{r}, X_{-r}\right\rangle \not \leq U_{r} .
$$

Clearly we can replace $g$ by $n_{w}$ since $X_{r}$ normalizes $U_{r}$ and $\left\langle X_{r}, X_{-r}\right\rangle$. So we are required to prove

$$
n_{w}^{-1} U_{r} n_{w} \cap\left\langle U_{r}, X_{r}, X_{-r}\right\rangle \not \leq U_{r} .
$$

Since $w(r) \neq \pm r$ we know that, for some $s \in\{r,-r\}$,

$$
n_{w} X_{s} n_{w}^{-1}<U_{r} .
$$

This implies (1) and so there exists a $p$-element in $G_{\mathfrak{L}}$ lying in

$$
\left\langle U_{r}, X_{r}, X_{-r}\right\rangle \backslash U_{r} .
$$

This element will normalize $U_{r}$ and so $G_{\mathfrak{L}} \geq B$. This is a contradiction.

Thus if there exists $g \in G_{\mathfrak{L}} \backslash\left(P_{\Pi \backslash K^{\prime}} \cap G_{\mathfrak{L}}\right)$ then we can take $g=u_{1} n_{w} u$ as before and $w(r)= \pm r$. In fact, just as before, we can without loss of generality assume that $g=x_{r}(t) n_{w} x_{r}(u)$.

Now suppose that for all $s$, adjacent fundamental roots of $r$, we have $w(s)$ in $\Phi^{+} \cup \Phi_{\Pi \backslash K}^{-}$. Since $G_{\mathfrak{L}}>L_{\Pi \backslash K}$ we can assume that $w(s)$ is positive for all fundamental roots not equal to $r$. But then, by [?, Theorem 2.2.2], $w=w_{r}$ or $w=1$ (see also [?, Lemma 13.1.3] for the twisted case). However $G_{\mathfrak{L}}$ also contains $n_{r}$ and so we can assume that $g=x_{ \pm r(t)} x_{r}(u)$. In this case though $g \in P_{\Pi \backslash K^{\prime}}$ which is a contradiction. 
Thus there exists an adjacent fundamental root of $r, s$ say, such that $w(s)$ is negative. Define $h:=g x_{s}(v) g^{-1}$. Clearly, as before, we can suppose that $h=x_{r}\left(v_{1}\right) n_{w_{1}} x_{r}\left(v_{2}\right)$.

Now observe that $g \in\left\langle X_{r}, X_{-r}\right\rangle N_{N}\left(\left\langle X_{r}, X_{-r}\right\rangle\right)$. Suppose that $h$ also lies in $\left\langle X_{r}, X_{-r}\right\rangle N_{N}\left(\left\langle X_{r}, X_{-r}\right\rangle\right)$. Then this would imply that

$$
x_{s}(v) \in\left\langle X_{r}, X_{-r}\right\rangle N_{N}\left(\left\langle X_{r}, X_{-r}\right\rangle\right) .
$$

This is clearly impossible, see [?, Corollary 8.4.4, Proposition 13.5.3].

Thus $h \notin\left\langle X_{r}, X_{-r}\right\rangle N_{N}\left(\left\langle X_{r}, X_{-r}\right\rangle\right)$. This implies that $w_{1}(r) \neq \pm r$. Furthermore since $w(s) \notin \Phi^{+} \cup \Phi_{\Pi \backslash K}^{-}, h \notin P_{\Pi \backslash K^{\prime}}$. Then we can apply the same argument to $h$ as we applied to $g$ above. This will lead us to conclude that $G_{\mathfrak{L}} \geq B$ which is a contradiction.

This leads to the following result:

Lemma 4.1. Suppose that $G$ is a Chevalley group with $G_{\alpha}=P_{\Pi \backslash r}$. Then

$$
U_{r} L_{\Pi \backslash K} \leq G_{\mathfrak{L}} \leq P_{\Pi \backslash K^{\prime}}
$$

Suppose alternatively that $G$ is a twisted group with $G_{\alpha}=P_{\mathfrak{P} \backslash J}$. Suppose further that $G \neq{ }^{2} \mathrm{~F}_{4}(q)^{\prime}$ and $G \neq{ }^{2} \mathrm{~A}_{n}(q)$, $n$ even. Then

$$
U_{J}^{1} L_{\mathfrak{P} \backslash K} \leq G_{\mathfrak{L}} \leq P_{\mathfrak{P} \backslash K^{\prime}}
$$

where $K=J \cup K^{\prime}$ and $K^{\prime}$ is the set of orbits of fundamental roots in $\mathfrak{P}$ which contain roots not orthogonal to some root in $J$.

We record the following lemma of Saxl:

Lemma 4.2. [?, Lemma 2.6] If $X$ is a group of Lie type of characteristic $p$ acting on cosets of a maximal parabolic subgroup, then there is a unique subdegree which is a power of $p$ except where $X$ is one of $\mathrm{PSL}_{n}(q), \mathrm{P}_{2 m}^{+}(q)$ (m odd) or $\mathrm{E}_{6}(q)$.

For the moment let us exclude the exceptions listed in these two lemmas; then Lemma 4.2 suggests that if $G_{\alpha}=P_{r}$ then $G_{\mathfrak{L}}$ contains some $G$-conjugate of $L_{r}$. This clearly contradicts Lemma 4.1. Note also that even in the listed exceptions of Lemma 4.2 many of the maximal parabolic subgroups have a unique subdegree which is a power of $p$.

\subsection{The twisted exceptions}

We consider the exceptional cases listed in Lemma 4.1. In fact we need only consider when $\left(G, G_{\alpha}\right)$ is one of $\left({ }^{2} \mathrm{~A}_{n}(q),{ }^{\wedge}\left[q^{\frac{n^{2}+4 n}{4}}\right] \cdot \mathrm{GL}_{\frac{n}{2}}\left(q^{2}\right)\right), n$ even; or $\left({ }^{2} \mathrm{~F}_{4}(q)\right.$, $\left.\left[q^{22}\right]: \mathrm{GL}_{2}\left(q^{2}\right)\right), q^{2}=2^{1+2 a}, a \geq 1$. 